\title{
Right-left and up-down are equally discriminable in the absence of directional words
}

\author{
RUTH H. MAKI \\ North Dakota State University, Fargo, North Dakota 58105
}

\begin{abstract}
Maki, Grandy, and Hauge (1979) found that right-left discriminations were not more difficult than up-down discriminations when no directional words were used. However, Farrell (1979) found that right-left discriminations took longer than up-down discriminations in a nonverbal go/no-go task. Two experiments were conducted to isolate the reasons for the different results. No directional words were used in either experiment, and there was no difference between response times to right-left and up-down stimuli in several different conditions. While there may have been some subtle procedural difference between Farrell's procedures and ours, generally, right-left discriminations do not seem to take longer than up-down discriminations when directional words are not used.
\end{abstract}

There is now ample evidence that adults have more difficulty in telling right from left than in telling up from down when the directional words are involved in the discrimination task (Farrell, 1979; Maki, Grandy, \& Hauge, 1979; Maki, Maki, \& Marsh, 1977). There is also a fair amount of evidence that young children have more difficulty with right-left than with up-down stimuli in tasks in which directional words are not used (Reudel \& Teuber, 1963; Wohlwill \& Wiener, 1964). However, it is much less clear that left-right discriminations are more difficult than up-down discriminations for adults when the words are not involved in the task. In experiments in our laboratory (Maki et al., 1979), the words were eliminated from a discrimination task by having subjects learn to pair directional stimuli with arbitrary labels, viz., arrows and letters. After learning was completed, subjects participated in a reaction time (RT) experiment in which the directional stimuli were paired with the letters; subjects decided whether the pairings were correct or incorrect. In two experiments, there was absolutely no difference between the horizontal and vertical stimuli when the arbitrary letters were used. However, horizontal stimuli did take longer than vertical stimuli when directional words were used and subjects decided whether the direction of the stimuli matched that described by the word.

In contrast to these findings, Farrell (1979, Experiment 3) found that right-left stimuli took longer than up-down stimuli in a go/no-go discrimination task that did not involve the directional

I am grateful to Cheryl Grandy and Verlin Hinsz for testing the subjects in Experiment 2 . Send reprint requests to Ruth $\mathrm{H}$. Maki, Department of Psychology, North Dakota State University, Fargo, North Dakota 58105. words. He presented V-shaped arrows that pointed up, down, right, or left; during a block of trials, subjects pushed a button in response to only one of the stimuli. Each subject responded to all stimuli in a counterbalanced order. On the average, rightleft stimuli took longer than up-down stimuli. Because no directional words were involved in Farrell's task, the results are inconsistent with our conclusion (Maki et al., 1979) that the right-left stimuli themselves pose no difficulty to subjects but that the labels are the source of people's difficulties with right and left.

There are several procedural differences between the tasks used by Farrell (1979) and the tasks used in our experiments. Farrell's task was a simple go/ no-go discrimination with directional stimuli, whereas our task involved responding to pairings of directional stimuli and letters. The pairing task may have been too complex to allow detection of small differences between the right-left and up-down stimuli. In our experiments, stimuli remained on the screen until the subject responded; in Farrell's experiments, stimuli were presented for $100 \mathrm{msec}$. Our subjects had consistent pairings of stimuli and letters; Farrell's subjects changed their responses to stimuli during the experiment. For example, some of Farrell's subjects responded only to the right stimulus during the first 50 trials of right and left stimuli, then they responded only to the left stimulus for $\mathbf{5 0}$ trials. For the final 100 trials, up and down stimuli were presented and the subjects responded first to one stimulus for 50 trials and then to the other one for 50 trials. Perhaps right and left stimuli were no more difficult than up and down stimuli during the first block of trials, but it was more difficult to change from responding to one horizontal stimulus to 
responding to the other than to change within the vertical dimension. If so, an overall average would yield longer RTs for horizontal than for vertical stimuli. Experiment 1 was designed to determine whether these procedural differences might account for the different results found by Maki et al. (1979) and by Farrell (1979). The task was a go/no-go discrimination task much like that used by Farrell.

\section{EXPERIMENT 1}

\section{Method}

Design and Subjects. Forty-eight volunteers from introductory psychology courses at North Dakota State University were randomly assigned to four groups. Two groups of subjects saw all stimuli for $100 \mathrm{msec}$. For the other two groups, a response terminated the stimulus to which the subject was supposed to respond and the other stimulus was terminated after $2 \mathrm{sec}$. Two groups of subjects were presented with the right-left stimuli first and two groups were presented with the up-down stimuli first. Within these orders, half of the subjects responded to one stimulus first and half responded to the other stimulus first.

Procedure. Stimuli were presented on a DECscope VT52 terminal controlled by an Automated Data Systems 1800E minicomputer. The stimuli consisted of nine asterisks arranged in a $\mathrm{V}$ shape. The point of each $\mathrm{V}$ was up, down, right, or left. The two sides of the $\mathrm{V}$ formed a 90-deg angle for both the horizontal and vertical stimuli. Because of the spacing constraints on the terminal display, the length of the sides of the arrows was $3.5 \mathrm{~mm}$ for the right-left and $3.25 \mathrm{~mm}$ for the up-down stimuli. Half of the subjects first saw a random sequence of right-left stimuli and half saw a random sequence of up-down stimuli first. Each subject was instructed to respond to only one of the two stimuli by saying "yes" into a microphone attached to a Lafayette voice-activated relay. Care was taken not to mention the directional words in the instructions. Subjects were shown examples of the stimuli on index cards and instructed to respond to one but not to the other. Twenty practice trials were given, followed by 50 trials during which subjects responded to one stimulus. Three other sets of 50 trials followed during which subjects responded to each of the three other directional stimuli. Each set of trials was preceded by an instruction displayed on the screen which indicated the stimulus to which subjects were to respond during the next set of trials. Ten practice trials were given following each new instruction.

For the response-terminated group, the stimulus to which the subject was to respond was displayed until a response was made or until $2 \mathrm{sec}$ had elapsed. The other stimulus was displayed for $2 \mathrm{sec}$. In the brief-exposure condition, both stimuli were displayed for $100 \mathrm{msec}$. In order to eliminate subjects' reliance on environmental cues, the RT task was performed in a dark room. Sessions lasted approximately $20 \mathrm{~min}$.

\section{Results and Discussion}

Mean RTs in milliseconds are shown in Table 1. These were determined by finding each subject's mean RT for horizontal and for vertical stimuli and averaging across subjects. A 2 by 2 by 2 mixed-design analysis of variance was conducted with stimulus duration (brief exposure vs. response terminated) and order (right-left vs. up-down first) as betweensubjects variables and stimulus orientation as a within-subjects variable. Only the interaction of Stimulus Duration by Order by Orientation was
Table 1

Mean RTs (Milliseconds) in the Go/No-Go Discrimination Task as a Function of Exposure Duration, Orientation, and Order

\begin{tabular}{lccccc}
\hline & \multicolumn{2}{c}{ Brief Exposure } & & Response Terminated \\
\cline { 2 - 3 } & $\begin{array}{c}\text { Right- } \\
\text { Left }\end{array}$ & $\begin{array}{c}\text { Up- } \\
\text { Down }\end{array}$ & $\begin{array}{c}\text { Right- } \\
\text { Left }\end{array}$ & $\begin{array}{c}\text { Up- } \\
\text { Down }\end{array}$ \\
\hline Right-Left First & 392 & 392 & & 376 & 333 \\
Up-Down First & 370 & 373 & & 346 & 373 \\
Mean RT & 381 & 382 & & 361 & 353 \\
\hline
\end{tabular}

significant $[F(1,44)=6.26$, MSe $=1,072.24, p<.05$, the significance level to be used for all statistical tests]. To understand the interaction, simple main effects analyses were conducted for each stimulus duration group. For the brief-exposure group (the replication of Farrell's procedures), no effects were significant $(F s<1)$. For the response-terminated group, there was an interaction of Orientation by Order $[\mathrm{F}(1,22)=16.54, \mathrm{MSe}=882.24]$. As shown in Table 1, subjects responded faster to stimuli that were presented second, regardless of whether they were oriented vertically or horizontally. Thus, there was a significant practice effect in this condition but there was no hint of slower responding to right-left than to up-down stimuli. The overall error rate was less than $1 \%$, so errors were not analyzed.

Farrell's (1979) finding of longer RTs to rightleft than to up-down stimuli in a go/no-go discrimination task was not replicated. Because there was no suggestion of a difference between the overall right-left and up-down RT means in the present experiment, it is unlikely that Farrell found his results because changing responding from one stimulus to its opposite is more difficult with horizontal than with vertical stimuli. Likewise, the brief stimulus duration used by Farrell does not explain why he fould slower responding to horizontal stimuli and we did not in earlier experiments.

\section{EXPERIMENT 2}

Another possible difference between Farrell's (1979, Experiment 3) experiment and our experiments (Experiment 1 and Maki et al., 1979) might be the instructions. In our experiments, we were careful to avoid the use of directional words in the instructions. Sample stimuli were pointed to in the instructions, so the directional words themselves were not mentioned. While it is unclear from Farrell's procedures whether the words "right," "left," "up," and "down" were mentioned in the instructions, it may be a source of the difference between our studies and his. Farrell's subjects may have translated the directional stimuli into the directional words during the RT task. In Farrell's Experiment 1, he showed that naming horizontal stimuli took longer than naming vertical stimuli, so, 
if such a translation process did occur during the go/no-go discrimination task, RTs to horizontal stimuli would take longer than RTs to vertical stimuli. Because no directional words were ever used in our instructions, subjects may have responded to the stimuli themselves and not translated them into the directional words.

In Experiment 2, the pairing task used earlier was employed. Half of the subjects learned to associate right-left and up-down stimuli with letter labels, while the other half learned to associate the words "right," "left," "up," and "down" with the letter labels. Then all subjects participated in an RT task involving the letter-arrow pairings. Those subjects who learned the letter-word pairings may have found it necessary to translate the arrow into the directional word before responding to the letter-arrow pairs. Such a process should be similar to subjects translating the arrows into directional words as a result of instructions that use directional words. If horizontal stimuli take longer than vertical stimuli in the word learning condition, then such a translation could explain why Farrell found orientation effects in the go/no-go task while we found no such effects.

\section{Method}

Design and Subjects. Twenty-four subjects were randomly assigned to two learning groups. The word group learned directional words paired with letters; the arrow group learned directional arrows paired with letters. In the RT task, the design was a 2 by 2 by 2 mixed factorial with group as a betweensubjects factor and orientation of the arrows (horizontal or vertical) and truth as within-subjects factors.

Procedure. Stimuli were presented on the DECscope terminal controlled by an Automated Data Systems 1800E minicomputer. Subjects first learned the direction-letter pairs. Two of four sets of consonants (S-T, J-K, X-Y, and P-Q) were randomly selected for each subject. Each set was paired with either the horizontal or the vertical stimuli. Subjects in the word group learned a letter as a response to each directional word (right, left, up, and down). Subjects in the arrow group learned a letter as a response to arrows that pointed up, down, right, or left ( $\uparrow$, $\downarrow, \rightarrow, \leftarrow)$. The arrows were constructed of 11 asterisks. Learning was by a study-test procedure with each directional stimulus (word or arrow) presented above its letter on the terminal screen for $2 \mathrm{sec}$. After all four pairs had been presented, each stimulus was presented alone and the subject typed the appropriate letter on the terminal keyboard. This procedure continued until the subject had typed the correct responses on five consecutive test trials.

In the RT portion of the experiment, all subjects responded to arrow-letter pairs. A directional arrow was presented above one of the four letters on the terminal screen. Subjects in the word group were instructed to decide whether the orientation of the arrow was consistent with the directional word that had been paired with that letter. Subjects in the arrow group were instructed to decide whether the arrow-letter pair was the same as one they learned during the learning phase of the experiment. Subjects indicated their response by pressing one button for correct pairs (true) and another button for incorrect pairs (false). The two buttons were mounted $2.54 \mathrm{~cm}$ apart. For half of the subjects the two buttons were oriented at a 315-deg angle in front of the subject and for the other half the orientation of the response buttons was $45 \mathrm{deg}$. Half of the subjects used their right index fingers for both responses and half used their left index fingers. Twenty practice trials preceded 128 data collection trials. Half of the trials involved horizontal stimuli and half involved vertical stimuli. Half were true and half were false. On all false trials, the incorrect letter was the other member of the letter pair so than an arrow was paired with the other letter from the same dimension (e.g., an arrow pointing upward was paired with the "down" letter). For the RT portion of the experiment, the only illumination in the room was provided by a desk lamp behind the DECscope, so environmental right-left cues were not very salient.

\section{Results and Discussion}

Subjects in the arrow condition took an average of 8.05 trials to the criterion of five successive correct; subjects in the word condition took 17.58 trials to criterion. This difference was short of significance $[\mathrm{F}(1,22)=3.72, \mathrm{MSe}=145.45, \mathrm{p}<.10]$. Subjects in the arrow group made a mean total of 2.83 errors to vertical stimuli and 2.25 errors to horizontal stimuli. Subjects in the word group made a mean total of 10.0 errors to vertical stimuli and 10.83 errors to horizontal stimuli. In a mixed-design analysis of variance, the group effect yielded $F(1,22)=2.99$, $\mathrm{MSe}=249.1$, which was not significant. The effect of orientation and the Orientation by Group interaction were not significant $(\mathrm{Fs}<1)$. In terms of learning, then, the word group tended to take longer to meet criterion, but there were no differences in learning to pair letters with horizontal and with vertical stimuli.

The RT and error data are presented in Table 2. A 2 by 2 by 2 mixed-design analysis of variance of the RT data indicated that true RTs were faster than false RTs $[F(1,22)=7.21, \mathrm{MSe}=.014]$ and that group and orientation interacted $[F(1,22)=4.30$, MSe $=.042]$. A simple main effects analysis indicated that orientation was not significant for either the word or the arrow group. Therefore, the interaction must have occurred because there was a tendency for faster RTs to vertical than to horizontal stimuli

Table 2

Mean RTs (Seconds) and Proportion Errors (E) for the Arrow and Word Learning Groups in Experiment 2

\begin{tabular}{|c|c|c|c|c|c|c|c|c|}
\hline \multirow[b]{3}{*}{ Truth } & \multicolumn{4}{|c|}{ Arrow Group } & \multicolumn{4}{|c|}{ Word Group } \\
\hline & \multicolumn{2}{|c|}{ Right-Left } & \multicolumn{2}{|c|}{ Up-Down } & \multicolumn{2}{|c|}{ Right-Left } & \multicolumn{2}{|c|}{ Up-Down } \\
\hline & RT & $\mathrm{E}$ & RT & $\mathbf{E}$ & RT & $\mathbf{E}$ & RT & $\mathbf{E}$ \\
\hline $\begin{array}{l}\text { True } \\
\text { False }\end{array}$ & $\begin{array}{l}1.188 \\
1.252\end{array}$ & $\begin{array}{l}.044 \\
.010\end{array}$ & $\begin{array}{l}1.173 \\
1.196\end{array}$ & $\begin{array}{l}.036 \\
.029\end{array}$ & $\begin{array}{l}1.144 \\
1.194\end{array}$ & $\begin{array}{l}.091 \\
.029\end{array}$ & $\begin{array}{l}1.247 \\
1.366\end{array}$ & $\begin{array}{l}.057 \\
.050\end{array}$ \\
\hline Mean RT & 1.220 & & 1.184 & & 1.170 & & 1.306 & \\
\hline
\end{tabular}


in the arrow group but the reverse trend in the word group. This interaction was exactly the opposite as what was predicted by the translation hypothesis. That is, it was expected that subjects in the word group would be likely to translate the arrows into the directional words and show longer RTs to horizontal stimuli but that the arrow group, in which translatin was less likely, would not. It is possible that such translation occurred early in the session but dropped out with practice. To test this, RTs were also analyzed for the first half of the trials. In the word condition, RTs were 1.407 and 1.224 for vertical and horizontal trials, respectively; in the arrow condition, RTs were 1.218 and 1.288 for vertical and horizontal trials. The analysis of variance produced exactly the same effects as the overall analysis. Thus, if the translation process occurred early in the session and then dropped out, it must have done so very quickly, because none of the data indicated that horizontal stimuli took longer than vertical stimuli in the word condition.

The proportion errors are also shown in Table 2 . An analysis of variance indicated that direction and truth interacted $[\mathrm{F}(1,22)=5.04, \mathrm{MSe}=.002]$. For true responses, there was a trend toward more errors to horizontal than to vertical stimuli, but this was reversed with false responses.

The results of this experiment failed to support the view that the wording of the instructions could explain why Farrell (1979) found effects of stimulus orientation on RT in the go/no-go task while we did not. In the present experiment, directional words were used in the learning phase, a manipulation that should have been much stronger than simply using directional words in instructions. Yet, we found no effect of stimulus orientation either in the word or in the arrow condition. Thus, Farrell probably did not find that horizontal stimuli took longer than vertical stimuli because his instructions involved directional words.

\section{GENERAL DISCUSSION}

Both experiments indicated that right-left stimuli took no longer in RT tasks than up-down stimuli when the directional words were not used. These results are consistent with our earlier experiments (Maki et al., 1979) but they are not consistent with the results found by Farrell (1979, Experiment 3).
Farrell's use of brief stimulus exposures and the requirement that subjects change from responding to one stimulus to responding to the opposite stimulus cannot explain why he found an orientation effect and we did not. Farrell's stimuli were presented via a tachistoscope, while ours were presented via a cathode-ray-tube terminal. It is unlikely that this was the source of the different findings because we conducted an earlier experiment that was similar to Experiment 2 of this report except that we used a projection tachistoscope. In that experiment we found no difference between right-left and up-down stimuli when the directional words were absent (Maki, Note 1). Thus, the particular method of presentation was probably not responsible for the different results. Either there is some very subtle difference between the procedures used by Farrell and the procedures used in all of our experiments or the results of Farrell's Experiment 3 represent a statistical Type I error.

The bulk of the evidence is consistent with our earlier conclusion that horizontal stimuli themselves pose no difficulty relative to vertical stimuli for adults but the words right and left are more difficult than the words up and down. How these words came to be difficult when the stimuli themselves are not difficult continues to be an intriguing question.

\section{REFERENCE NOTE}

1. Maki, R. H. The role of label encoding in right-left as compared to up-down judgments. Paper presented at the meeting of the Midwestern Psychological Association, Chicago, 1977.

\section{REFERENCES}

Farrell, W. S., JR. Coding left and right. Journal of Experimental Psychology: Human Perception and Performance, 1979, 5, 42-51.

Maki, R. H., Grandy, C. A., \& Hauge, G. Why is telling right from left more difficult than telling above from below? Journal of Experimental Psychology: Human Perception and Performance, 1979, 5, 52-67.

Maki, R. H., Maki, W. S., \& Marsh, L. G. Processing locational and orientational information. Memory \& Cognition, $1977,5,602-612$.

Reudel, R. G., \& Teuber, H. L. Discrimination of direction of line in children. Journal of Comparative and Physiological Psychology, 1963, 56, 892-898.

Wohlwill, J., \& Wiener, M. Discrimination of form orientation in young children. Child Development, 1964, 35, 1113-1125.

(Received for publication June 13, 1979.) 